Revisión de tema

\title{
Metamateriales: principales características y aplicaciones
}

\author{
Luis M. Castellanos, Francisco López, Erick Reyes-Vera* \\ Facultad de Ingenierías, Instituto Tecnológico Metropolitano, Medellín, Colombia
}

\begin{abstract}
Resumen
Las propiedades electromagnéticas de todos los materiales existentes en la naturaleza pueden ser determinadas a partir dos parámetros, la permeabilidad magnética y la permitividad eléctrica, los cuales nos permiten caracterizar la respuesta de cualquier material cuando este interactúa con una onda electromagnética. En principio, no existe límite para el rango de valores que estos dos parámetros pueden tomar. Por tanto, es posible diseñar y construir a voluntad materiales con características de respuesta electromagnética específicas no encontradas en la naturaleza. Estos materiales fabricados en el laboratorio reciben el nombre genérico de metamateriales, y entre ellos se encuentran los conocidos por LHM, así llamados porque los vectores de campo de las ondas electromagnéticas que viajan en su interior están relacionados por la regla de la mano izquierda. La característica distintiva de los LHM es que para ciertas bandas de frecuencia presentan índice de refracción negativo con modos propagativos posibles. Este fenómeno se presenta solamente si ambos parámetros $\mu$ y $\varepsilon$, dentro de dichas bandas de frecuencias son negativos simultáneamente. El propósito de este trabajo es presentar los principios y fundamentos de estos metamateriales de manera que despierte el interés de lectores no especializados. (C) 2016. Acad. Colomb. Cienc. Ex. Fis. Nat.
\end{abstract}

Palabras clave: Metamateriales; Anillos resonadores; Ondas electromagnéticas.

Metamaterials: main features and applications

\begin{abstract}
Electromagnetic properties of all materials existing in nature can be determined from two parameters, the magnetic permeability and the electrical permittivity, which allow us to characterize the response of any material when this interacts with an electromagnetic wave. In principle, there is no limit to the range of values that can be taken by these two parameters. Therefore, it is possible to design and construct materials with specific characteristics of electromagnetic response not found in nature will. These materials manufactured in the laboratory received the generic name of Metamaterials, and among them the well-known by LHM, are so called because the vectors of field of electromagnetic waves travelling in the interior are related by the rule of the left hand. The distinctive characteristic of the LHM is that for certain bands of frequency they present negative index of refraction with possible propagative modes. This phenomenon appears only if both parameters $\mu$ and $\varepsilon$, within these bands of frequencies are simultaneously negative. The purpose of this paper is to present the principles and foundations of these metamaterials so that it wakes up the interest of not specialized readers. (C) 2016. Acad. Colomb. Cienc. Ex. Fis. Nat.
\end{abstract}

Key words: Metamaterials; Ring resonators; Electromagnetic waves.

\section{Introducción}

El principio físico fundamental en el caso de un metamaterial consiste en "sustituir" átomos por microestructuras fabricadas en el laboratorio, las cuales al igual que los átomos, presenten actividad electromagnética, es decir, tienen una función dieléctrica $\varepsilon(\omega)$ y una magnética $\mu(\omega)$. El tamaño de las microestructuras debe asemejarse a las de un átomo, a fin de permitir la propagación de una onda electromagnética (OEM) en el interior del material sin que se genere difracción. De esta manera, cuando la OEM avanza en el interior del metamaterial lo hace como si estuviera avanzando en un material homogéneo. Este comportamiento es asegurado por la condición de homogeneidad efectiva (Caloz \& Itoh, 2005), la cual compara la longitud de la onda que se propaga con el tamaño de la celda unitaria del metamaterial,

$$
\frac{\lambda}{4} \gg a \text {. }
$$

Cuando una onda electromagnética se propaga en el interior de un medio material, los parámetros $\mu(\omega)$ y $\varepsilon$ $(\omega)$, dependen de la frecuencia de la onda incidente. Sin

\footnotetext{
*Correspondencia:

Erick Reyes-Vera, erickreyes@itm.edu.co

Recibido: 11 de marzo de 2016

Aceptado: 28 de junio de 2016
} 
embargo, si tenemos en cuenta la frecuencia $\omega_{p}$ del plasmon asociado al medio material, esta dependencia podría incluso inhibir la propagación de la onda en el interior del medio (Mark A. Heald, 1994), pues recordemos que el vector de onda puede ser expresado como

$$
k=\frac{\omega}{\mathrm{c}} \sqrt{1-\frac{\omega_{p}^{2}}{\omega^{2}}}
$$

donde $k$ es la magnitud del vector de onda $\hat{k}$, $\omega$ es la frecuencia de la onda, $\omega_{p}$ es la frecuencia plasmónica y $c$ es la velocidad de la luz. De la ecuación (2), es claro que la onda se propaga solo si se cumple que la frecuencia de la onda es mayor a la frecuencia plasmónica $\left(\omega>\omega_{p}\right)$. Adicionalmente, es importante recordar que $k=\frac{\omega}{\mathrm{c}} n=\frac{\omega}{\mathrm{c}} \sqrt{\mu \varepsilon}$, es decir, todo el comportamiento electromagnético (EM) del metamaterial y su respuesta cuando dicho material interactúa con una onda electromagnética (OEM) depende del índice de refracción $n$ y por lo tanto de su permitividad eléctrica $\varepsilon$ y su permeabilidad magnética $\mu$. Pero, ¿qué son en realidad los parámetros $\varepsilon$ y $\mu$ ? De acuerdo con la teoría electromagnética, estos son una medida de la capacidad de almacenar energía eléctrica y magnética que tiene determinada estructura. De lo anterior, es evidente que para aumentar la actividad magnética y eléctrica de un material, es necesario incrementar su capacidad eléctrica y magnética, es decir, diseñar estructuras que puedan almacenar energía eléctrica y magnética de manera más eficiente que las conocidas hasta este momento. Donde tradicionalmente para llevar a cabo dicha función han sido implementados arreglos de capacitores e inductores. Uno de los sistemas que nos brinda la posibilidad de tener simultáneamente estos dos mecanismos de almacenamiento son los circuitos LC. Dichos circuitos, poseen una frecuencia de resonancia asociada dependiente de la capacitancia e inductancia del sistema, donde la frecuencia de resonancia está dada por $\omega=\frac{1}{\sqrt{L C}}$.

Uno de los mecanismos más simples que nos permiten emular los circuitos LC es la implementación de SRR (Split Ring Resonator, por sus siglas en inglés) los cuales permiten incrementar la capacitancia e inductancia de forma simultánea de un sistema. En aras de dar mayor claridad al lector, dicha estructura es esquematizada en la Figura 1. Nótese cómo un rectángulo con una abertura de ancho $d$ replica un circuito LC, donde la inductancia $L$ está distribuida en toda la extensión geométrica del cuadrado y la capacitancia puede ser modificada dependiendo del tamaño de la abertura $d$. Dicha estructura fue el resultado de diversos estudios realizados en la década de los 90’s, en la cual investigadores de diferentes grupos de investigación se preocuparon por proponer diseños que permitieran lograr los objetivos mencionados antes. En la siguiente sección presentamos una de las estructuras que se ha constituido en la piedra angular para el estudio, diseño y construcción de metamateriales, la cual funcionan muy bien especialmente en el rango de las microondas.

\section{Anillos resonadores}

En 1999, Pendry y su grupo sugieren que ciertas configuraciones de medios conductores no magnéticos podrían tener respuesta magnética considerablemente fuerte cuando son sometidas a la presencia de un campo electromagnético (J. B. Pendry, Holden, Robbins, \& Stewart, 1999a). En particular, predijeron que estas configuraciones tendrían permeabilidad magnética negativa en un rango determinado y finito de frecuencias. La estructura propuesta consistía en un doble anillo con las aberturas orientadas en sentidos opuestos como se muestra en la Figura 2.

La novedad de la estructura propuesta por Pendry radicaba en la disposición de las aberturas de los anillos y tiene la siguiente explicación: Cuando la OEM se propaga en el interior del material excita o induce una corriente eléctrica (flecha en color azul) en la misma dirección en ambos anillos (sentido horario en ambos anillos de la Figura 2). Adoptando la convención usual en ingeniería, según la cual los portadores de carga en la corriente son positivos, entonces la abertura del anillo exterior dispone la acumulación de cargas positivas en la mitad superior del anillo, y por defecto, de cargas negativas en la mitad inferior del anillo. En el anillo interior, la abertura dispone las cargas positivas en la parte inferior y, por defecto, las negativas en la parte superior. El resultado final, como puede notarse a simple vista, es la creación de dos condensadores conectados en serie, cada uno
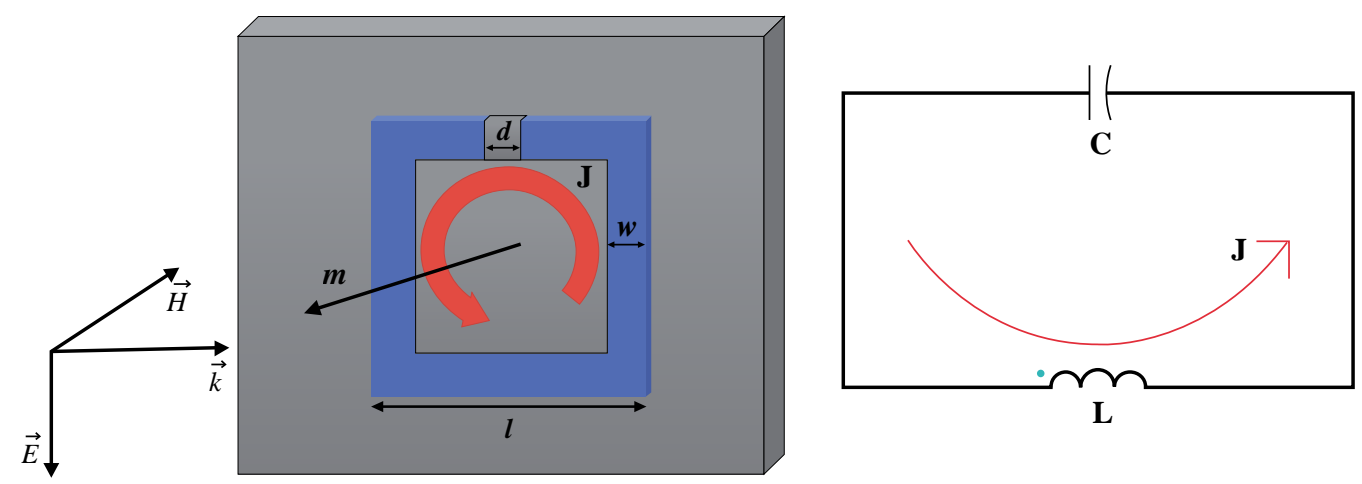

Figura 1. Un rectángulo con abertura de ancho la apertura funciona como capacitor, mientras la inductancia está distribuida en toda la estructura rectangular. Se puede representar por un circuito LC. 
de ellos con una apreciable capacidad eléctrica e inductancia magnética distribuida. El número de anillos utilizados para construir la estructura solo está limitado, en principio, por la condición de homogeneidad efectiva.

Una vez aceptado que un anillo con abertura equivale a un circuito $L C$, podemos construir estructuras con capacitancias e inductancias apreciablemente mayores, agregando anillos concéntricos. Toda la estructura sería el resultado de conectar eléctricamente los circuitos elementales equivalentes de cada resonador. Las propiedades electromagnéticas de los SRR

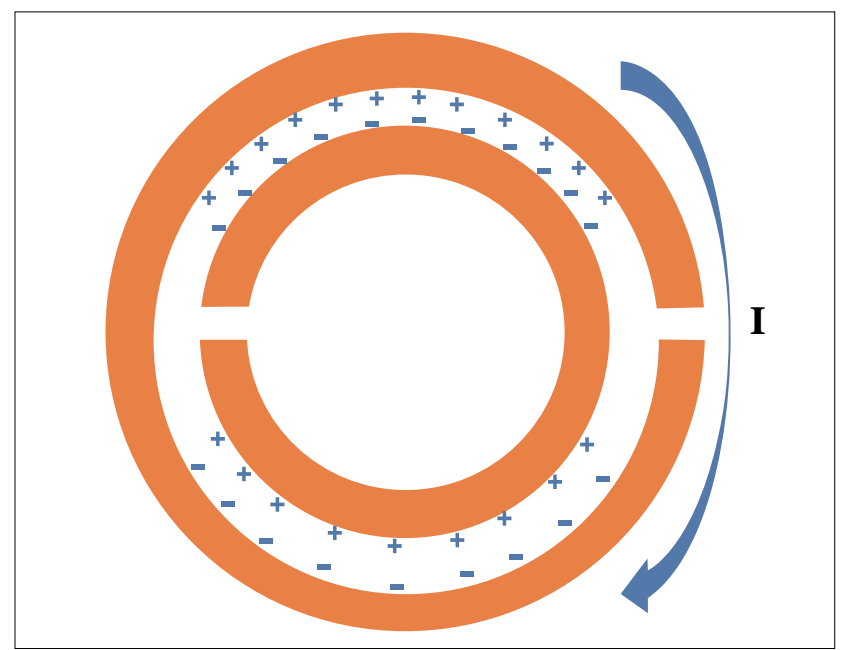

Figura 2. Anillos resonadores, elemento propuesto por J. Pendry: La disposición de las aberturas es fundamental para lograr un efecto de capacitancia eléctrica distribuida. El número de anillos que componen la estructura aumenta la inductancia.

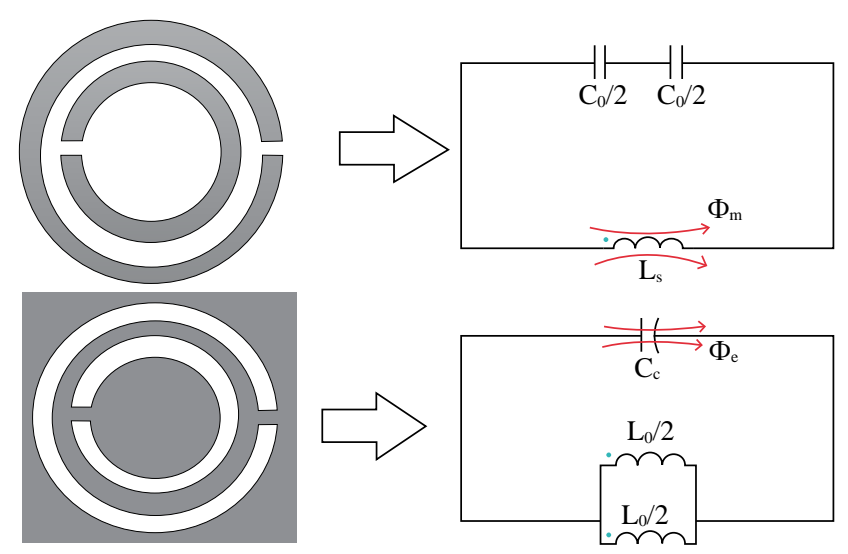

Figura 3. En la parte superior se muestra una estructura compuesta de dos resonadores SRR (propuesta por Pendry) y a la derecha su modelo circuital equivalente En la parte inferior se muestra el CSRR y su circuito equivalente a la derecha. Nótese el cambio en las conexiones de los capacitores y las inductancias. Un SRR se construye imprimiendo sobre un substrato dieléctrico los anillos conductores. Por el contrario un CSRR se obtiene "canalizando" sobre un substrato conductor los anillos, donde había anillos conductores y substrato dieléctrico ahora hay anillos dieléctricos y substrato conductor. han sido ampliamente analizadas en (Marqués, Medina, \& Rafii-El-Idrissi, 2002; Marques, Mesa, Martel, \& Medina, 2003) y un modelo circuital equivalente para resonadores SRR y CSRR (Complementary Split ring Resonator, por su sigla en inglés) fue presentado en el 2005 por (Baena, et al., 2005), como se ilustra en la Figura 3. Si las perdidas por resistencia de los anillos no son considerables, el espesor de los mismos no tiene influencia alguna y se observa un comportamiento dual (Falcone, et al., 2004), es decir los SRR se comportan esencialmente como dipolos magnéticos resonantes susceptibles a ser excitados con campos magnéticos axiales (paralelos al eje perpendicular del plano del anillo), mientras los CSRR se comportan como dipolos eléctricos con la misma frecuencia de resonancia también susceptible a ser excitados con un campo eléctrico axial.

\section{Cálculo de la Permeabilidad Magnética de un Resonador}

Es posible obtener la permeabilidad magnética para un resonador simple con abertura a partir de la teoría elemental de circuitos. Para este caso en particular no existe capacitancia distribuida y por lo tanto todo el efecto capacitivo es debido a la abertura de ancho $d$ presente en el anillo resonador. Así, cuando una OEM viaja con dirección y polarización como se ilustra en la Figura 1 (es decir, el campo magnético $\vec{H}(t)$ oscilando en la dirección perpendicular al plano del SRR.) induce una corriente que al acoplarse con el campo eléctrico oscilante $\vec{E}$ puede entrar en resonancia con este. Entonces podemos utilizar las leyes de Kirchhoff para obtener el balance de energías del sistema. Para ello, basta con representar los SRR o CSRR como circuitos $L C$ (ver Figura 3), donde el potencial inducido por las oscilaciones de $\vec{H}(t)$ lo representariamos por una fuente de voltaje alterno y así, el balance de energía en un instante de tiempo $t$ cualquiera está dado por:

$$
V_{\text {inducido }}=-\dot{\Phi}_{B}=L \dot{I}+\frac{1}{C} \int_{0}^{t} I d t
$$

Derivando con respecto al tiempo obtenemos

$$
-\ddot{\Phi}_{B}=L \ddot{I}+\frac{1}{C} I \text {. }
$$

Si además asumimos que la sección transversal del campo magnético es uniforme sobre toda el área del SRR, $\Phi_{B}$ representa su flujo a través de dicha área. Por otro lado, es razonable asumir que la corriente inducida $I$ y el campo inductor $\vec{H}(t)$ tienen la misma dependencia armónica temporal, y recordando que la densidad de campo magnético y el vector de magnetización están dados por $\overrightarrow{\boldsymbol{B}}=\mu_{0} \overrightarrow{\boldsymbol{H}}$ y $\overrightarrow{\boldsymbol{M}}=n \overrightarrow{\boldsymbol{m}}$ y respectivamente, donde $\overrightarrow{\boldsymbol{m}}=I l^{2} \overrightarrow{\boldsymbol{u}}_{n}$. En este caso específico se tiene que $n$ es el número de espiras de corriente por unidad de volumen, $m=I l^{2}$ es el momento dipolar magnético y $l$ el lado de la espira.

Después de operar y organizar términos es posible obtener una expresión para la permeabilidad magnética dada por (5)

$$
\mu_{r}(\omega)=\left(1+\frac{F \omega^{2}}{\left(\omega_{L C}^{2}-\omega^{2}\right)}\right),
$$

Donde $F=N \frac{l^{2} t}{V}$, y $N$ el número total de resonadores en el volumen de la celda. De lo anterior, tenemos entonces que todos los resonadores presentaran una permeabilidad 
magnética dada por la ecuación (5) donde el factor $F$ es un parámetro característico de cada resonador. La Figura 4 nos muestra la respuesta característica obtenida para un anillo resonador como el ilustrado en la Figura 1. Claramente para este caso particular la permeabilidad magnética puede tomar valores negativos para un rango de frecuencias entre $4.77 \mathrm{GHz}$ y $6.77 \mathrm{GHz}$ aproximadamente, donde dicho rango puede ser alterado si modificamos la geometría de la estructura empleada.

\section{M etamateriales con índice de refracción negativo}

La posible existencia de materiales con índice de refracción negativo fue teóricamente explorada por primera vez en 1968, por el físico ruso Víctor Veselago (Veselago, 1968). Estas sustancias deberían tener permitividad y permeabilidad negativas. Veselago en ese momento las nombró zurdas o materiales izquierdos (LH: Left Handed en inglés), dado que el trío que forman el vector de propagación, el campo eléctrico y el campo magnético siguen una ley de mano izquierda a diferencia de los dieléctricos convencionales que siguen una ley de mano derecha. Como consecuencia, en los medios LH el vector de Poynting viaja en dirección contraria a la dirección de propagación de la onda como se puede ver en la Figura 5b, al contrario de los medios RH (Right Handed Material, por sus siglas en inglés) donde el vector de Poynting viaja en la misma dirección de propagación de la onda (Figura 5a).

Esto se puede verificar fácilmente a partir de las ecuaciones de Maxwell y teniendo presente que los campos $\vec{E}(\mathbf{r}, t)$ y $\overrightarrow{\boldsymbol{B}}(\mathbf{r}, t)$, son de carácter ondulatorio:

$$
\begin{array}{r}
\vec{\nabla} \times \overrightarrow{\boldsymbol{E}}=-\frac{\partial \overrightarrow{\boldsymbol{B}}}{\partial t} \Rightarrow \overrightarrow{\boldsymbol{\kappa}} \times \overrightarrow{\boldsymbol{E}}=\omega \mu \overrightarrow{\boldsymbol{H}} \\
\vec{\nabla} \times \overrightarrow{\boldsymbol{H}}=-\frac{\partial \overrightarrow{\boldsymbol{D}}}{\partial t} \Rightarrow \overrightarrow{\boldsymbol{\kappa}} \times \overrightarrow{\boldsymbol{E}}=\omega \boldsymbol{G} \overrightarrow{\boldsymbol{H}}
\end{array}
$$

Nótese que si $\epsilon$ y $\mu$ son ambos positivos los vectores $\vec{E}, \overrightarrow{\boldsymbol{H}}$, y $\overrightarrow{\boldsymbol{\kappa}}$ forman lo que se conoce como un triplo de mano derecha y al medio activo a través del que se propagan se le denomina RHM. Si por el contrario si $\epsilon$ y $\mu$ son simultáneamente negativos, estos formaran un triplo de mano izquierda, y al medio se le denomina LHM.

Veselago predijo algunos efectos producidos por un medio zurdo, entre los cuales se destacan:

1. La velocidad de fase y velocidad de grupo son antiparalelas.

2. Inversión del efecto Doppler.

3. Inversión de la radiación de Vavilov- Cerenkov.

4. Inversión de las condiciones de contorno relativas a los componentes normales de los campos magnéticos y eléctricos en el área de contacto entre un medio zurdo y un medio diestro.

5. Inversión de la ley de Snell.

6. Índice de refracción negativo en la superficie de contacto entre un medio zurdo y un medio diestro.

7. Transformación del punto fuente en un punto imagen en un medio zurdo.

8. Intercambio de los efectos de divergencia y convergencia en lentes convexas y cóncavas, respectivamente, cuando están fabricadas con un medio zurdo.

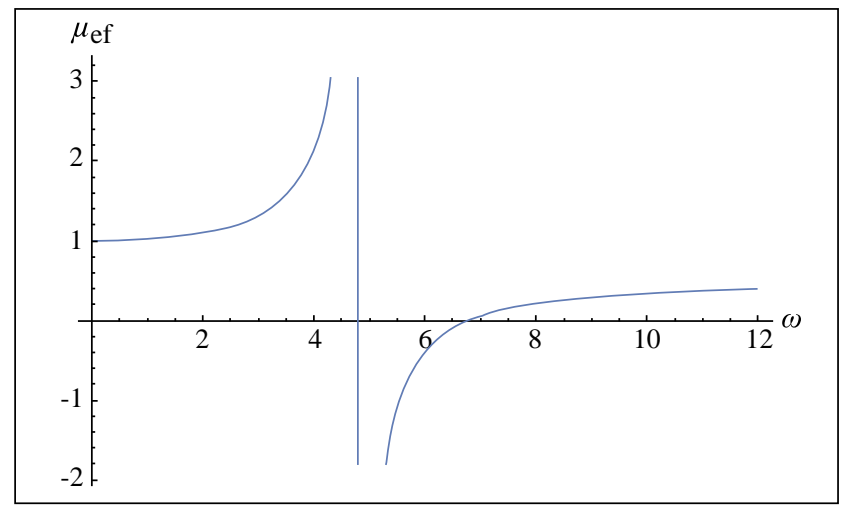

Figura 4. Respuesta magnética del SRR de la figura 1 con $l=$ $2 \mathrm{~cm}, w=0,4 \mathrm{~cm}, d=0,5 \mathrm{~mm}, t=30 \times 10^{-6} \mathrm{~m}$. El valor de la frecuencia de resonancia $\omega_{L C}=\frac{1}{\sqrt{L C}}=\frac{c_{0}}{l \sqrt{k}} \sqrt{\frac{d}{W}}$, (hemos utilizado $C=\frac{\varepsilon A}{d}=\frac{\varepsilon W t}{d}=\frac{k \varepsilon_{0} W t}{d}$ y además $L=\mu_{0} \frac{l^{2}}{t}$ ).

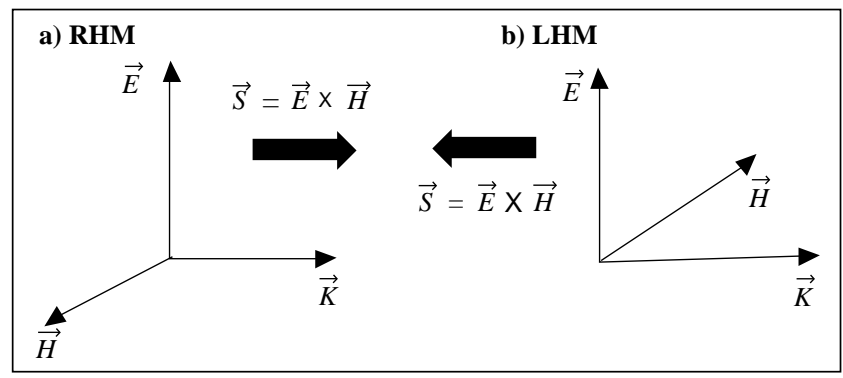

Figura 5. En un RHM el vector de Poynting $\overrightarrow{\boldsymbol{s}}$ viaja en la misma dirección que el vector de propagación de onda $\overrightarrow{\boldsymbol{\kappa}}$. En cambio para el caso de un LHM, dichos vectores tienen direcciones opuestas.

9. Fenómenos de tipo resonante ante plasmones.

Veselago solo describió el potencial e hipotético comportamiento de sustancias zurdas, dado que para la fecha del estudio (1968) se desconocía material alguno que pudiera presentar estas características. Por esta razón ningún experimento se pudo realizar y quedó solo como un postulado teórico hasta el año 1999.

\section{Construcción de los primeros materiales LH M}

En 1999 se construyó el primer material LHM. No se trataba de ninguna sustancia, sino de una estructura artificial lo suficientemente pequeña para ser considerada homogénea, es decir, una estructura que cumplía la condición de homogeneidad efectiva. Dicha estructura fue concebida por Smith y colaboradores (D. R. Smith, Padilla, Vier, Nemat-Nasser, \& Schultz, 2000) y se basó enteramente en los trabajos de Pendry, (J. B. Pendry, et al., 1999a; J. B. Pendry, Holden, Robbins, \& Stewart, 1999b; J. Pendry, Holden, Stewart, \& Youngs, 1996). Entre 1996 hasta 1998 el grupo dirigido por J.B. Pendry propuso y demostró que con alambres delgados, los cuales llamaremos en adelante TW (Thin wires, por su sigla en inglés) se podían obtener permitividades negativas para el rango de las microondas ( $\mathbf{J}$. B. Pendry, et al., 1999b; J. Pendry, et al., 1996). 
Inmediatamente después del workshop de 1999 "Electromagnetics Crystal Structures", en Laguna Beach, donde Pendry presentó por primera vez la estructura de anillo resonador, Smith y colaboradores, quienes asistieron al evento, iniciaron un programa para construir el primer medio con índice de refracción negativo. La idea del equipo de Smith fue juntar las dos estructuras presentadas por Pendry para conseguir simultáneamente permeabilidad y permitividad negativas, lo que presentaría un índice de refracción $n$ real y, por lo tanto, permitiría propagación. El diseño de Smith consistió en construir por separado una estructura TW y otra SRR para el mismo rango de frecuencias y, posteriormente, combinarlas para la obtención de una estructura compuesta TW-SRR (D. Smith, Padilla, Vier, Nemat-Nasser, \& Schultz, 2000).

Adicionalmente, diversos trabajos de simulaciones numéricas mostraron que la localización espacial de los SRR con respecto a los alambres era de importancia crítica para construir una banda de propagación LHM. Los mejores resultados se obtuvieron cuando los alambres se colocaron entre los SRR, con los ejes de los alambres intersectando los ejes de los SRR. Esto llevó al grupo de Smith a concluir que tal configuración minimizaba la interacción entre los anillos y los alambres, permitiendo así una división clara entre las respuestas eléctrica (los alambres) y magnética (los SRR) (D. R. Smith, et al., 2000; Yen, et al., 2004). Cuando no se consideran pérdidas (lo cual se logra utilizando buenos conductores para la construcción de la estructura TW-SRR), dichas respuestas vienen dadas por

$$
\varepsilon(\omega)=1-\frac{\omega_{\mathrm{p}}^{2}}{\omega^{2}} ; \quad \mu_{r}(\omega)=\left(1+\frac{F \omega^{2}}{\left(\omega_{L C}^{2}-\omega^{2}\right)}\right)
$$

Para comprobar su naturaleza zurda se lanzó una onda electromagnética dentro de la banda de paso de ambas estructuras constitutivas, y se comprobó que los parámetros constitutivos $\varepsilon$ y $\mu$ presentaban valores simultáneamente negativos.

\section{Aplicaciones de los metamateriales}

Desde su descubrimiento, los metamateriales han sido involucrados en diversos campos de la ciencia y la ingeniería. Una de las áreas específicas que mayor interés ha tenido en su implementación ha sido el de las antenas, radio propagación y comunicaciones inalámbricas, debido entre otras cosas a que la implementación de estructuras metamateriales permitiría mejorar la respuesta eléctrica de antenas y filtros de radiofrecuencia. En efecto, se ha reportado el incremento del ancho de banda comparado con las antenas de microcinta tradicionales (L.-W. Li, Li, Soon Yeo, Mosig, \& Martin, 2011; Wang \& $\mathbf{L i}, 2006$ ), de la eficiencia de radiación de las antenas (Ouedraogo, Rothwell, Diaz, Fuchi, \& Temme, 2012), de la ganancia (Chaimool, Chung, \& Akkaraekthalin, 2010; Wu, et al., 2005) e incluso han sido implementados en los últimos años con el fin de minimizar las dimensiones de la antena, mientras simultáneamente se mantienen las propiedades eléctricas, algo que era imposible lograr con la tecnología tradicional. Este hallazgo sin duda ha sido uno de los puntos destacados de los metamateriales y su implementación en aplicaciones de comunicaciones inalámbricas, pues incluso se han reportado resultados en los cuales se ha logrado disminuir las dimensiones físicas hasta un $70 \%$, lo cual, es un gran resultado si tenemos en cuenta que la mayoría de dispositivos tecnológicos actuales requieren de sistemas electrónicos y de comunicación altamente compactos para mejorar la eficiencia (Fan-Yi Meng, Qun Wu, Bo-Shi Jin, Wang, \& Jian Wu, 2007). Esto ha permitido que las antenas basadas en esta tecnología sean fácilmente integradas en formatos de electrónica flexible o en dispositivos micro y nanométricas para llevar a cabo no sólo comunicaciones inalámbricas, sino también operaciones de sensado en tiempo real (Cheng, Senior, Kim, \& Yoon, 2011; Senior, et al., 2011; Xiaoyu Cheng, Jun Shi, Jao, Senior, \& Yong-Kyu Yoon, 2011). Es importante mencionar algunos trabajos recientes que analizan el comportamiento de antenas construidas con capas de materiales distribuidas de forma periódica y sobre sustratos FR4, los cuales son de bajo costo (Tauseef Asim \& Ahmed, 2015). Los autores utilizan la misma celda unitaria para fabricar una meta pantalla para la parte inferior y un súper sustrato para la parte superior del parche y así estudiar los efectos sobre la impedancia, el ancho de banda, la ganancia y los patrones de radiación.

Las áreas de sensores y filtros en RF son quizás las que más han empleado los metamateriales para mejorar el desempeño. Muestra de ello son los numerosos trabajos que han sido publicados por los principales grupos de investigación a nivel mundial (Ebrahimi, Withayachumnankul, Al-Sarawi, \& Abbott, 2014; Ebrahimi, et al., 2014; Rusni, Ismail, Alhawari, Hamidon, \& Yusof, 2014). Cabe destacar que estas aplicaciones no sólo se han restringido a la región de las microondas, sino que han trascendido a otras zonas del espectro electromagnético, lo cual sin lugar a dudas ha incrementado su interés. Muchos autores han empezado a demostrar su aplicabilidad en la región óptica (Falcone, et al., 2004; Linden, 2004; Linden, et al., 2006; Shelby, 2001).

Otras aplicaciones interesantes de los metamateriales conciernen a la generación de invisibilidad de superficies o zonas, ya que a través de dispositivos fabricados con estos materiales de permitividad y permeabilidad negativas, es posible ocluir una zona determinada de modo que un observador no pueda determinar si un objeto está presente en ella (Schurig, et al., 2006). Adicionalmente, en los últimos años aplicaciones relacionadas con almacenamiento de energía han sido exploradas por algunos grupos de investigación, los cuales han logrado demostrar que, al implementar paneles con este tipo de estructuras, se logra incrementar la eficiencia de conversión de energía gracias a que como se mencionó antes, este tipo de estructuras nos permiten modificar la capacitancia y por ende la capacidad de almacenamiento de energía (Almoneef \& Ramahi, 2015). 
Por último, en años recientes se ha explorado el uso de metamateriales y Meta superficies para el diseño y construcción de lentes especiales (súper lentes o híper lentes) con el fin de generar cambios que pueden revolucionar aspectos de la óptica convencional, principalmente en algunos aspectos asociados a aplicaciones ligadas a la holografía. Diversos grupos han demostrado que a través de la combinación de dieléctricos y metales es posible desarrollar lentes con mayor poder de focalización, de mayor calidad (se disminuyen aberraciones y efectos difractivos), mayor magnificación y simultáneamente más compactas que las lentes convencionales (Chen, Chang, \& Chen, 2016; Jacob, Alekseyev, \& Narimanov, 2006; R. Li, et al., 2015; Lipworth, et al., 2014) lo cual hace posible su integración en sistemas micro y nanométricos.

Recientemente, los autores han explorado el uso de metamateriales, específicamente en el diseño y la construcción de diferentes dispositivos de radiofrecuencia tales como antenas y resonadores tipo parche para su utilización como sensores o dispositivos para comunicaciones inalámbricas. Se han obtenido algunos resultados interesantes para la medición de la permitividad dieléctrica relativa de materiales acuosos y gaseosos con $\varepsilon<10$, usando una estructura de metamaterial basada en un resonador rectangular grabado en una antena de microcinta. Demostrando que este tipo de estructuras es altamente sensible a cambios en la permitividad del medio que la rodea, principalmente gracias al incremento en la capacitancia como se mencionó anteriormente (Domínguez, Cataño, \& Reyes, 2015). Adicionalmente, han estudiado los efectos generados en la respuesta eléctrica de antenas de microcinta cuando sobre el parche es grabado un arreglo de CSRR. Demostrando que es posible optimizar el acople de energía por parte de la antena, minimizar las perdidas por retorno y sintonizar la frecuencia de resonancia a través de la manipulación de los parámetros geométricos del arreglo, como lo son el periodo del arreglo y el tamaño de cada celda unitaria (Catano-ochoa, Senior, Lopez, \& Reyes-Vera, 2016).

\section{Conclusiones}

Hemos realizado una revisión general e introductoria sobre metamateriales, con el interés principal de mostrar qué es y cómo se entiende un metamaterial y cuáles son sus posibles aplicaciones. Hemos mostrado que la posibilidad de "fabricar materia" usando estructuras TW-SRR, con propiedades según requerimientos particulares, abre nuevos horizontes en el estudio del electromagnetismo aplicado y la ciencia de los materiales, que pueden llevar a nuevos e interesantes resultados. Adicionalmente se ha demostrado los grandes avances en ciertas áreas de la ingeniería y las ciencias gracias a la implementación de estas estructuras.

\section{Agradecimientos}

Los autores agradecen el apoyo financiero del Instituto Tecnológico Metropolitano a través del proyecto P13252.

\section{Conflicto de intereses}

Los autores declaran no tener conflicto de intereses.

\section{Referencias}

Almoneef, T., \& Ramahi, O. M. 2015. Split-ring resonator arrays for electromagnetic energy harvesting. Progress In Electromagnetics Research B, 62 (January): 167-180. doi:10.2528/PIERB15012506

Baena, J. D., Bonache, J., Martin, F., Sillero, R. M., Falcone, F., Lopetegi, T., Sorolla, M. 2005. Equivalent-circuit models for split-ring resonators and complementary splitring resonators coupled to planar transmission lines. IEEE Transactions on Microwave Theory and Techniques. 53 (4): 1451-1461. doi:10.1109/TMTT.2005.845211

Caloz, C., \& Itoh, T. 2005. Electromagnetic Metamaterials: Transmission Line Theory and Microwave Applications. (I. John Wiley \& Sons, Ed.) (First.). Hoboken, NJ, USA: John Wiley \& Sons, Inc. doi:10.1002/0471754323

Catano-ochoa, D., Senior, D. E., Lopez, F., \& Reyes-Vera, E. 2016. Performance Analysis of a Microstrip Patch Antenna loaded with an Array of Metamaterial Resonators. In IEEE International Symposium on Antennas and Propagation/ USNC-URSI National Radio Science (pp. 1-3). Fajardo, Puerto Rico: IEEE.

Chaimool, S., Chung, K. L., \& Akkaraekthalin, P. 2010. Simultaneous gain and bandwidths enhancement of a single-feed circularly polarized microstrip patch antenna using a metamaterial reflective surface. Progress In Electromagnetics Research B, 22: 23-37. doi:10.2528/ PIERB10031901

Chen, Y.-A., Chang, I.-L., \& Chen, L.-W. 2016. Spiral hyperlens with enhancements of image resolution and magnification. Journal of Modern Optics, 63 (11): 1029-1034. doi:10.108 0/09500340.2015.1117670

Cheng, X., Senior, D. E., Kim, C., \& Yoon, Y.-K. 2011. A Compact Omnidirectional Self-Packaged Patch Antenna With Complementary Split-Ring Resonator Loading for Wireless Endoscope Applications. IEEE Antennas and Wireless Propagation Letters, 10: 1532-1535. doi:10.1109/ LAWP.2011.2181315

Domínguez, M., Cataño, D., \& Reyes, E. 2015. Design a sensor of relative dielectric permittivity of a medium using an antenna microstrip with metamaterial structures. Actas de Ingeniería. 1 (1): 110-114.

Ebrahimi, A., Withayachumnankul, W., Al-Sarawi, S., \& Abbott, D. 2014. High-Sensitivity Metamaterial-Inspired Sensor for Microfluidic Dielectric Characterization. IEEE Sensors Journal. 14 (5): 1345-1351. doi:10.1109/JSEN. 2013.2295312

Falcone, F., Lopetegi, T., Laso, M. A. G., Baena, J. D., Bonache, J., Beruete, M., Sorolla, M. 2004. Babinet Principle Applied to the Design of Metasurfaces and Metamaterials. Physical Review Letters. 93 (19): 197401. doi:10.1103/ PhysRevLett.93.197401

Fan-Yi Meng, Qun Wu, Bo-Shi Jin, Wang, H.-L., \& Jian Wu. 2007. Comments on "Waveguide Miniaturization Using Uniaxial Negative Permeability Metamaterial. IEEE Transactions on Antennas and Propagation. 55 (3): 10161017. doi:10.1109/TAP.2007.891880

Jacob, Z., Alekseyev, L. V, \& Narimanov, E. 2006. Optical Hyperlens: Far-field imaging beyond the diffraction limit. Optics Express. 14 (18): 8247. doi:10.1364/OE.14.008247 
Li, L.-W., Li, Y.-N., Soon Yeo, T., Mosig, J. R., \& Martin, O. J. F. 2011. Addendum: "A broadband and high-gain metamaterial microstrip antenna.” Applied Physics Letters. 99 (15): 159901. doi:10.1063/1.3651481

Li, R., Guo, Z., Wang, W., Zhang, J., Zhou, K., Liu, J., Gao, J. 2015. Arbitrary focusing lens by holographic metasurface. Photonics Research. 3 (5): 252. doi:10.1364/PRJ.3.000252

Linden, S. 2004. Magnetic Response of Metamaterials at 100 Terahertz. Science, 306 (5700): 1351-1353. doi:10.1126/ science. 1105371

Linden, S., Enkrich, C., Dolling, G., Klein, M. W., Zhou, J., Koschny, T., Wegener, M. 2006. Photonic Metamaterials: Magnetism at Optical Frequencies. IEEE Journal of Selected Topics in Quantum Electronics, 12 (6): 1097-1105. doi:10.1109/JSTQE.2006.880600

Lipworth, G., Ensworth, J., Seetharam, K., Huang, D., Lee, J. S., Schmalenberg, P., ... Urzhumov, Y. 2014. Magnetic metamaterial superlens for increased range wireless power transfer. Scientific Reports. 4: 3642. doi:10.1038/srep03642

MarkA. Heald, J. B. M. 1994. Classical Electromagnetic radiation (Third Edit.). Mineola, New York: Dover Publications, Inc.

Marqués, R., Medina, F., \& Rafii-El-Idrissi, R. 2002. Role of bianisotropy in negative permeability and left-handed metamaterials. Physical Review B, 65 (14): 1-6. doi:10. 1103/PhysRevB.65.144440

Marques, R., Mesa, F., Martel, J., \& Medina, F. 2003. Comparative analysis of edge- and broadside- coupled split ring resonators for metamaterial design - theory and experiments. IEEE Trans. Ant. Propagat. 51 (10): 25722581. doi:10.1109/TAP.2003.817562

Ouedraogo, R. O., Rothwell, E. J., Diaz, A. R., Fuchi, K., \& Temme, A. 2012. Miniaturization of Patch Antennas Using a Metamaterial-Inspired Technique. IEEE Transactions on Antennas and Propagation. 60 (5): 2175-2182. doi:10.1109/ TAP.2012.2189699

Pendry, J. B., Holden, a. J., Robbins, D. J., \& Stewart, W. J. 1999a. Magnetism from conductors and enhanced nonlinear phenomena. IEEE Transactions on Microwave Theory and Techniques. 47 (11): 2075-2084. doi:10.1109/22.798002

Pendry, J. B., Holden, A. J., Robbins, D. J., \& Stewart, W. J. 1999b. Low frequency plasmons in thin-wire structures. Journal of Physics: Condensed Matter. 10 (22): 4785-4809. doi:10.1088/0953-8984/10/22/007

Pendry, J., Holden, A., Stewart, W., \& Youngs, I. 1996. Extremely Low Frequency Plasmons in Metallic Mesostructures. Physical Review Letters, 76 (25): 4773-4776. doi:10.1103/ PhysRevLett.76.4773

Rusni, I., Ismail, A., Alhawari, A., Hamidon, M., \& Yusof, N. 2014. An Aligned-Gap and Centered-Gap Rectangular Multiple Split Ring Resonator for Dielectric Sensing
Applications. Sensors, 14 (7): 13134-13148. doi:10.3390/ s140713134

Schurig, D., Mock, J. J., Justice, B. J., Cummer, S. A., Pendry, J. B., Starr, A. F., \& Smith, D. R. 2006. Metamaterial Electromagnetic Cloak at Microwave Frequencies. Science. 314 (5801): 977-980. doi:10.1126/science. 1133628

Senior, D. E., Cheng, X., Jao, P., Kim, C., Kim, J. K., \& Yoon, Y. 2011. Wireless passive sensing application using a cavity loaded evanescent mode half mode substrate integrated waveguide resonator. In 2011 16th International Solid-State Sensors, Actuators and Microsystems Conference (pp. 2014 2017). IEEE. doi:10.1109/TRANSDUCERS.2011.5969205

Shelby, R. A. 2001. Experimental Verification of a Negative Index of Refraction. Science. 292 (5514): 77-79. doi:10.1126/ science. 1058847

Smith, D., Padilla, W., Vier, D., Nemat-Nasser, S., \& Schultz, S. 2000. Composite Medium with Simultaneously Negative Permeability and Permittivity. Physical Review Letters. 84 (18): 4184-4187. doi:10.1103/PhysRevLett.84.4184

Smith, D. R., Padilla, W. J., Vier, D. C., Nemat-Nasser, S. C., \& Schultz, S. 2000. Composite medium with simultaneously negative permeability and permittivity. Physical Review Letters. 84 (18): 4184-4187. doi:10.1103/ PhysRevLett.84.4184

Tauseef Asim, M., \& Ahmed, M. 2015. Metamaterial Inspired Microstrip Antenna Investigations Using Metascreens. International Journal of Antennas and Propagation. 2015, 1-9. doi:10.1155/2015/236136

Veselago, V. 1968. The Electrodynamics of substances with simultaneously negative values of $\varepsilon$ and $\mu$. Soviet Physics Uspekhi. 10 (4): 509-514.

Wang, L., \& Li, J. L. 2006. A Novel Metamaterial Microstrip Antenna of Broadband and High-Gain. In Proceedings of ISAP2012 (pp. 806-809). Nagoya, Japan.

Wu, B.-I., Wang, W., Pacheco, J., Chen, X., Grzegorczyk, T. M., \& Kong, J. A. 2005. A study of using metamaterials as antenna substrate to enhance gain. Progress In Electromagnetics Research. 51: 295-328. doi:10.2528/ PIER04070701

Xiaoyu Cheng, Jun Shi, Jao, P., Senior, D. E., \& Yong-Kyu Yoon. 2011. Reconfigurable split ring resonator array loaded waveguide for insitu tuning. In 2011 IEEE International Symposium on Antennas and Propagation (APSURSI) (pp. 2947-2950). IEEE. doi:10.1109/APS.2011.5997146

Yen, T. J., Padilha, W. J., Fang, D. N., Vier, D. C., Smith, D. R., Pendry, J. B., ... Zhang, X. 2004. Terahertz Magnetic Response from Artificial Materials. Science. 303 (2004): 1494-1496. doi:10.1017/CBO9781107415324.004 